\title{
COMPARATIVE PERFORMANCE OF WATCHES WITH ELINVAR AND WITH STEEL HAIRSPRINGS
}

\author{
By Ralph E. Gould
}

\section{ABSTRACT}

The performance of watches having the usual cut, bimetallic balance wheels and steel hairsprings is compared with that of watches having uncut, monometallic balance wheels and elinvar hairsprings. The latter combination of vibrating assembly is a new application intended to improve the general performance of watches.

Twenty watches of each type, 10 each of 2 makes, were given performance tests at temperatures encountered in ordinary use. The temperature-rate errors of the watches having the new vibrating assembly were less than those for watches having the ordinary assembly, and instead of the usual parabolic curve, a curve approaching a straight line was obtained. Marked improvement in performance at temperatures within the usual temperature range 5 to $35 \mathrm{C}$ is indicated by the use of the new assembly.

The new assembly almost entirely overcomes the effects of magnetism so that, after the watch is removed from a magnetic field, the rate is not subject to fluctuations experienced with the ordinary assembly.

There is no evidence of any marked difference in performance of the two types of watches in different positions or for isochronism.

\section{CONTENTS}

I. Introduction . .

II. Effects of changes in temperature

III. Effects of changes in position

IV. Effects of magnetism

V. Isochronism

\section{INTRODUCTION}

The rate ${ }^{1}$ of a watch is affected by change in temperature to the same degree as is the free period of its vibrating assembly, which consists of the balance wheel with its hairspring and staff. In the usual design of the assembly, a steel hairspring is used. The effect of its change in stiffness with change of temperature upon the free period of the assembly is compensated by a change in moment of inertia of the balance wheel. The rim of the balance wheel is bimetallic (brass and steel) and is cut at 2 diametrically opposite points (fig. 1) so that a change in shape may take place as a result of unequal expansion of the 2 materials.

Watches with a new form of vibrating assembly have recently been placed on the market. In this assembly the hairspring is made of a nickel-steel alloy known as "elinvar" which has a temperature coefficient of elasticity which is very nearly 0 within the usual temperature range (5 to $35 \mathrm{C}$ ). With this hairspring an uncut, monometallic

1 Rate or daily rate is the number of seconds lost or gained in 24 hours. 
balance wheel (fig. 1), of nonmagnetic material, is used in place of the cut, bimetallic balance wheel.

Tests were made to determine the comparative performance of watches equipped with the usual vibrating assembly and of those equipped with the new assembly. Twenty watches of each type and
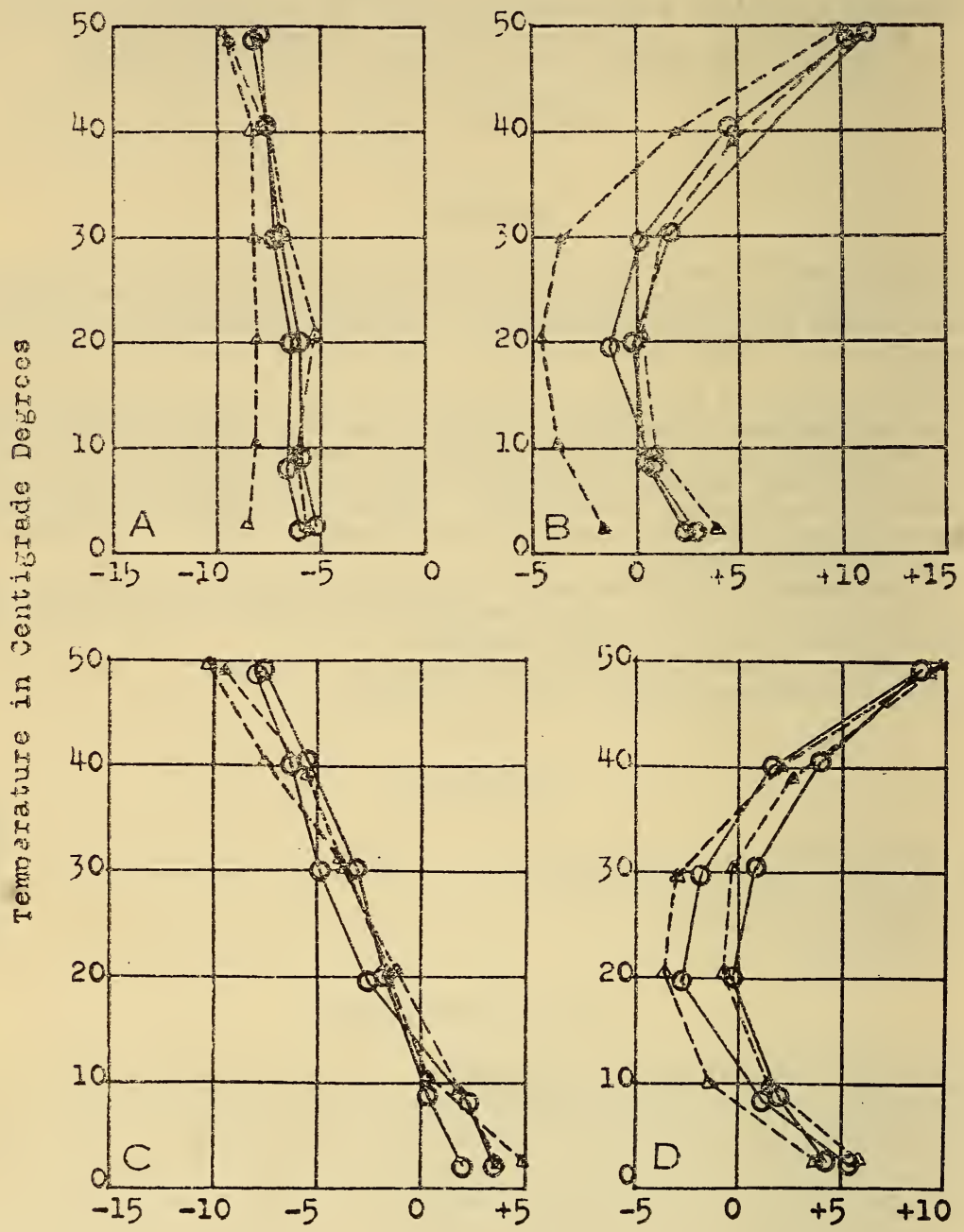

Deily Rate in Seconds

FigURE 2.-Temperature-rate curves.

$\mathrm{A}$ and $\mathrm{C}$. Watches having monometallic balance wheels and elinvar hairsprings. $\mathrm{B}$ and $\mathrm{D}$. Watches having bimetallic balance wheels and steel hairsprings. Each diagram is the composite curve for 10 similar watches of the same make. A and B represent watches of one make, while $\mathbf{C}$ and $\mathbf{D}$ represent those of another make.

of two different makes were tested at different temperatures, and in various positions. The effect of isochronism on these watches was determined. Three watches of each type were placed momentarily in magnetic fields of known intensity, and the rates determined after subjection to each field strength. 


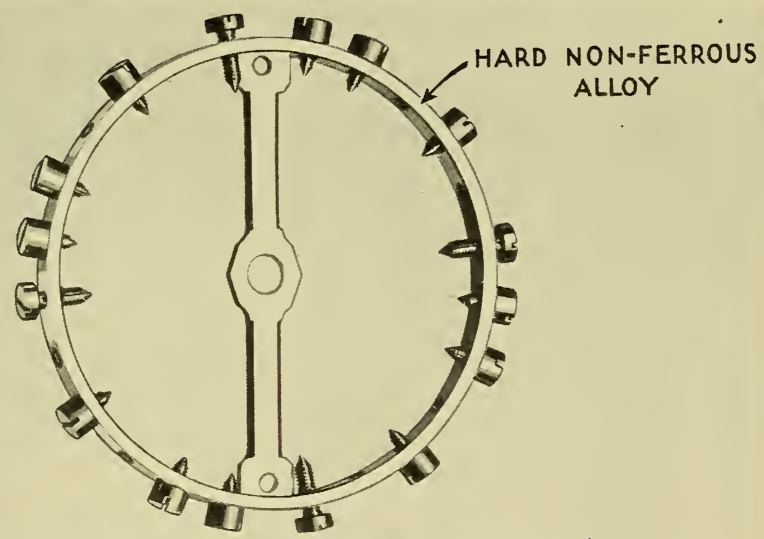

MONOMETALLIC BALANCE WHEEL (NOT CUT)

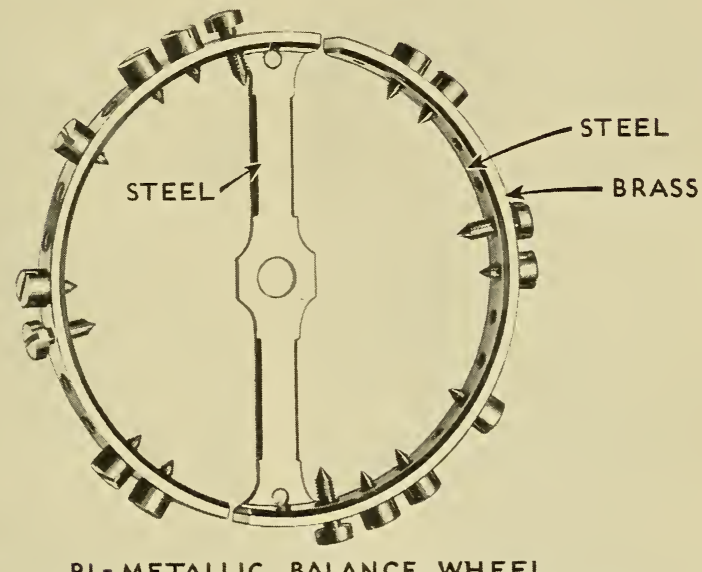

BI-METALLIC BALANCE WHEEL

FIGURE 1.-The new and old balance wheels. 


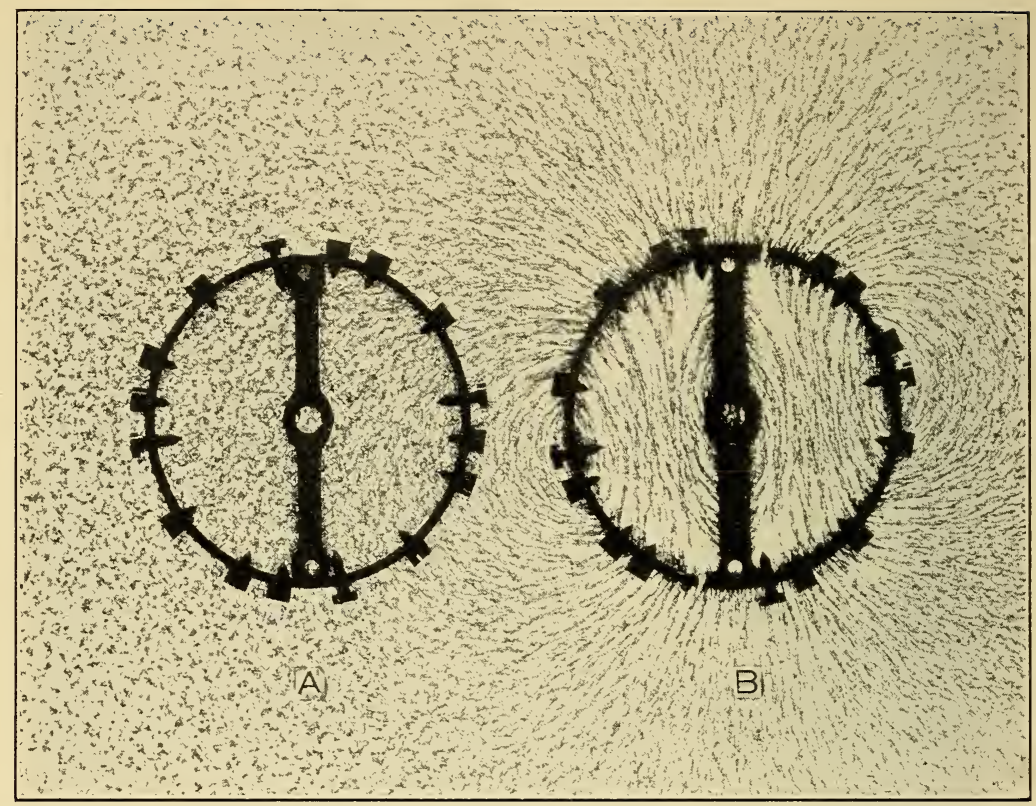

FIGURE 4.-Magnetic condition of the balance wheels after removal from a magnetic field.

A, Monometallic balance wheel; B, bimetallic balance wheel.

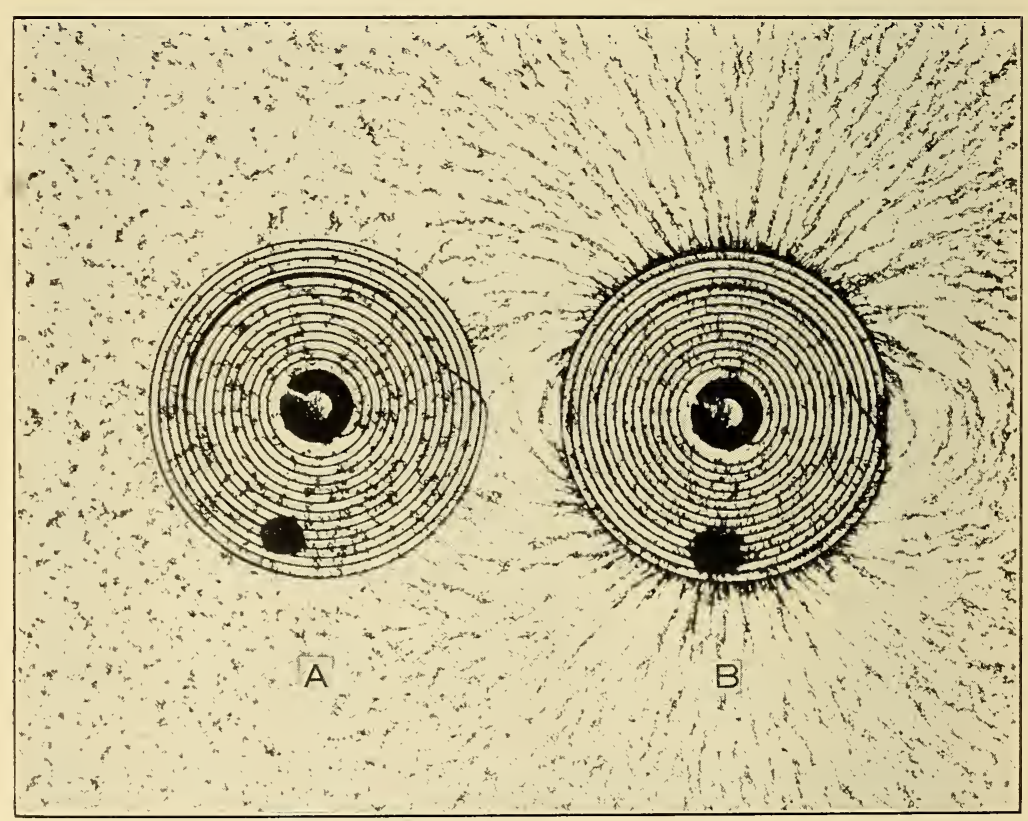

FIGURE 5.-Magnetic condition of the hairsprings after removal from a magnetic field.

A, Elinvar hairspring; B, steel hairspring. 


\section{EFFECTS OF CHANGES IN TEMPERATURE}

The effect of changes in temperature on the daily rates of 20 watches of each of the 2 types was determined. The watches were placed in a testing chamber in a horizontal, dial-up position and were simultaneously subjected to 2 temperature cycles from 0 to 50 to $0 \mathrm{C}$. The temperature was varied by steps of approximately $10 \mathrm{C}$. The watches were kept at each temperature for 2 days, and the daily rate was taken as the mean of the rates for the 2 days. Whenever the temperature was changed, the rate for that day was not used in computing the mean rate. Figure 2 shows the results of this test.

An inspection of the curves shows that the temperature-rate curves for watches with the ordinary vibrating assembly are parabolic in form, while those for watches with the new assembly are approximately straight lines. The slope of these straight lines, or the change in rate per degree $\mathrm{C}$, indicates the degree of temperature compensation of the watch. Because of the parabolic form of the curve, watches using the bimetallic balance wheel and steel hairspring show a considerable difference between the middle temperature $(20 \mathrm{C})$ rate and those at the high $(35 \mathrm{C})$ and low $(5 \mathrm{C})$ temperatures. With the new balance wheel and hairspring these differences are much reduced and indicate that with careful adjustment they may be practically eliminated.

The curves for the second cycle (triangles) do not coincide with those for the first cycle (circles), but have an appreciable displacement. Examination was made of the shellac which helps to hold the pallet jewels ${ }^{2}$ in their mountings and of the oil used in lubricating the watch. While the shellac showed a slight softening at temperatures above $45 \mathrm{C}$, there was no evidence of shifting of the jewels. The oil on several of the watches showed some signs of deterioration or gumming.

\section{EFFECTS OF CHANGES IN POSITION}

The 40 watches, mentioned above, were tested to determine whether or not any improvement in uniformity of rate in different positions resulted from the use of the new assembly. To eliminate any effect of temperature variations, the test was conducted at a constant temperature of $20 \mathrm{C}$. The watches were mounted firmly in holders on a frame which could be turned about different axes. The axes used were (1) one parallel to the hand staff of the watch, (2) one parallel to the 9-3 line of the dial, and (3) one parallel to the 12-6 line of the dial. The pendant in all cases was at the 12 point.

The rotation about an axis parallel to the hand staff was begun with the watches vertical, 12 up, dial front. The frame, with the watches, was rotated to the left through a complete revolution in steps of 15 degrees a day.

The rotation about an axis parallel to the 9-3 line was begun with the watches horizontal, dial up, 12 back. The frame was rotated forward in steps of 15 degrees a day.

The rotation about an axis parallel to the 12-6 line was begun with the watches horizontal, dial up, 12 back. The frame was rotated to the left in steps of 15 degrees a day.

${ }^{2}$ Pallet jewels are the jewels which serre as rolling contact surfaces in transmitting the action of the balance wheel to the escape wheel. 
Daily rates were computed for each position of the watches and, because of close agreement in performance of the two makes of watches, composite curves (fig. 3) were plotted only for each axis of rotation. The general forms of the curves conform to those expected on the basis of the ideas advanced by Kleinlein ${ }^{3} \mathrm{Gribi}^{4}{ }^{4}$ and others, and depend upon the relative locations of the inner and outer pinning points of the hairspring. From the forms of these curves it appears that no material change in performance with change of position results from the use of the new vibrating assembly.

\section{EFFECTS OF MAGNETISM}

The accuracy of the ordinary watch having a bimetallic balance wheel and a steel hairspring is greatly affected by magnetism. When

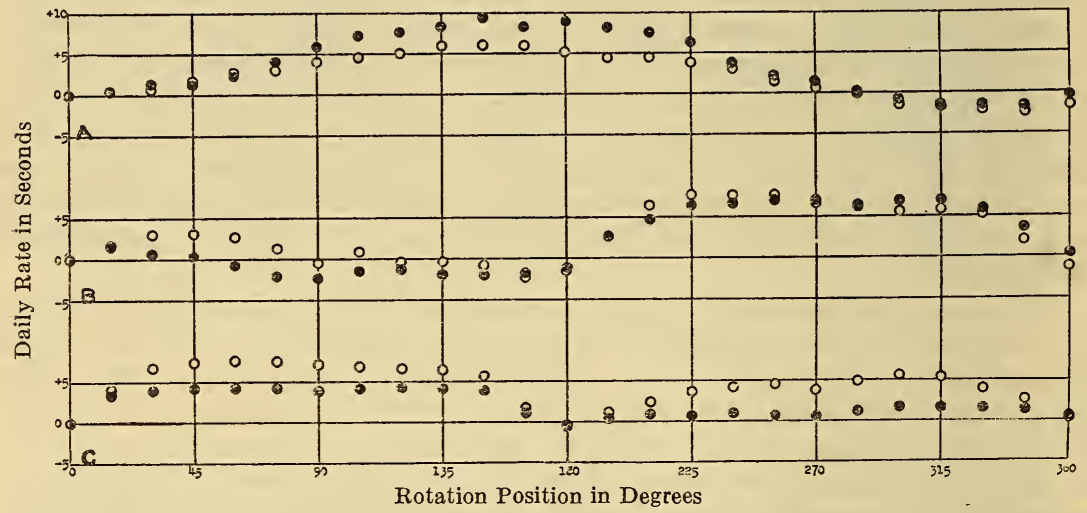

Figure 3.-Position-rate curves.

A. Rotation on an axis parallel to the hand staff; B. Rotation on an axis parallel to the 9-3 line; C. Curve for rotation on an axis parallel to the 12-6 line. The circles are composite values for 20 watches of 2 makes having monometallic balance wheels and elinvar hairsprings. The dots are composite values for 20 watches of 2 makes having bimetallic balance wheels and steel hairsprings.

placed in a strong magnetic field the steel hairspring and the balance arm become permanently magnetized; the hairspring is drawn out of place, sometimes even touching the balance arm; and the watch performs very erratically. The only remedy is a thorough demagnetizing of the watch.

Since elinvar has a temperature coefficient of elasticity which is nearly 0 within the usual temperature range, a cut bimetallic balance wheel is not needed for temperature compensation and an uncut wheel of nonmagnetic material may therefore be used. Also the elinvar hairspring, although it can be magnetized, does not retain its magnetism when the watch is removed from the magnetic field, and the performance of the watch is not permanently changed.

The magnetic conditions representative of the two types of balance wheels and hairsprings, after having been subjected to a magnetic field, are shown in figures 4 and 5 .

The rates of three watches of each type were determined after being subjected to magnetic fields of known strengths. These fields were

${ }^{3}$ W. J. Kleinlein, Practical Balance and Hairspring Work, and Rules and Practice of Adjusting Watches. W. J. Kleinlein, Comstock Building, Columbus, Ohio.

i Theo. Gribi, Practical Course in Adjusting. Jewelers' Circular Publishing Co., New York, N.Y., 1901. 
produced by a solenoid for which the field strength at the center is given by the formula:

$$
H=K I
$$

in which

$H=$ field strength in oersteds ${ }^{5}$

$K=$ an experimentally determined constant, in this case 88.5

$I=$ current in amperes

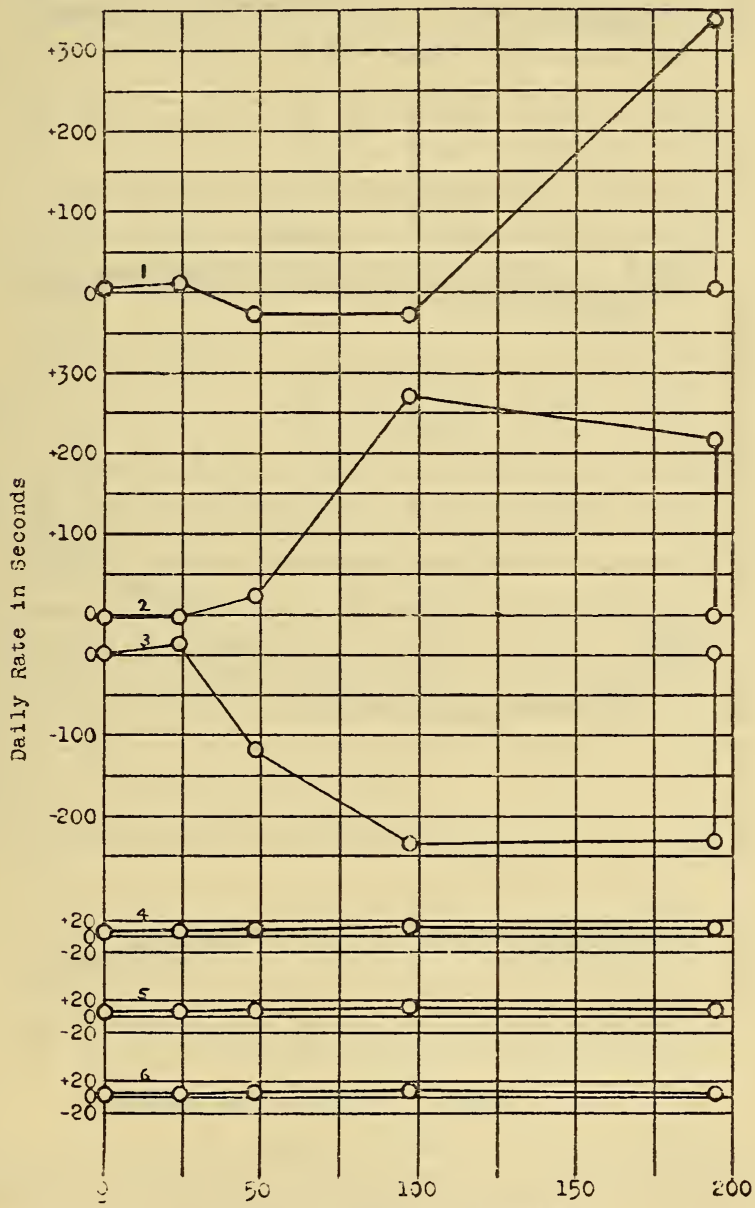

Field Strength in Cersteds

FIGURE 6.-Performance after subjecting watches to a magnetic field.

Curves 1, 2, and 3 are for watches having cut bimetallic balance wheels and steel hairsprings. Recovery of the original rate after demagnetization is shown by the last circle on the right, near the zero line. Curves

4,5 , and 6 are for watches having uncut, monometallic balance wheels and elinvar hairsprings.

The field strengths used were $24.3,48.2,97.4$, and 194.7 oersteds. $^{6}$

${ }^{8} \mathrm{~A}$ field strength of 1 oersted produces in empty space a magnetic induction of 1 gauss (sometimes called 1 line of force per square centimeter).

6 An idea of the relative values of the field strengths used may be gained from the fact that in the vicinity of Washington, D.C., the horizontal component of the earth's magnetic field is of the order of 0.2 oersted and the magnetic field close to an electric locomotive is approximately 50 oersteds. 
Before placing the watches in the magnetic field, their daily rates were obtained. They were then placed in the magnetic field of lowest strength for a few seconds, after which the daily rates were again obtained. Without demagnetizing, this procedure was repeated for increasing values of the magnetic field strength. After determining the daily rate for the highest field strength used, the watches were

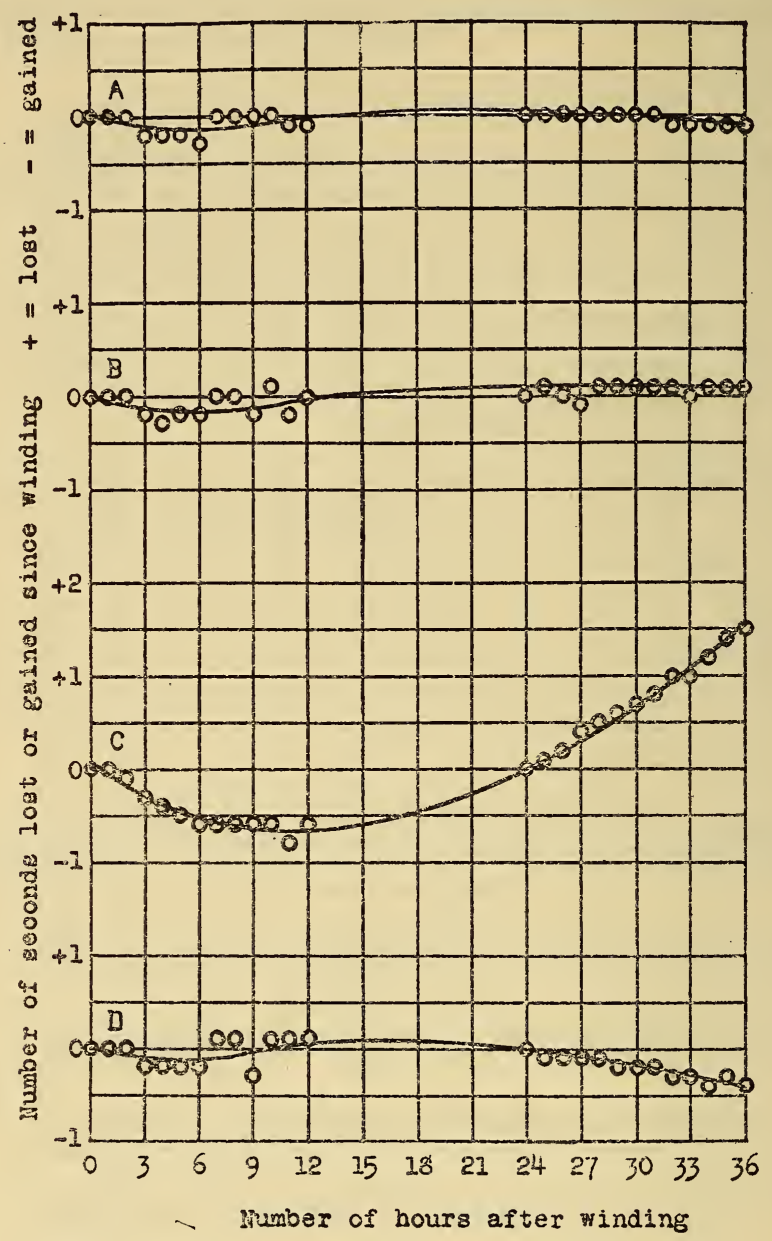

FIgURE 7.-Isochronal rate variation curves.

Curves $\mathrm{A}$ and $\mathrm{B}$ are for watches of one make. Curves $\mathrm{C}$ and $\mathrm{D}$ are for watches of another make. Curves $\mathrm{A}$ and $\mathrm{C}$ are for watches having uncut, monometallic balance wheels and elinvar hairsprings. Curves $\mathrm{B}$ and $\mathrm{D}$ are for watches having cut, bimetallic balance wheels and steel hairsprings.

demagnetized by placing them in an alternating magnetic field of approximately 165 oersteds.

The daily rates of these watches after each change of field strength are shown in figure 6 . These curves show a wide variation in performance for the three watches of the ordinary type, while they show practically no change in rate for watches having the new vibrating assembly. 
The new balance wheel, therefore, is a decided improvement over the old one in overcoming the effect of magnetic fields in which it may happen to be temporarily placed.

\section{ISOCHRONISM ${ }^{7}$}

Very few watches maintain an absolutely uniform rate as the mainspring unwinds. If rate be plotted against time after winding, true isochronism may be represented by a straight line drawn from the point representing the 24 -hour rate to the 0 point. If the variation from this line of the rates observed at different intervals after winding be plotted, on the same basis as for the aforementioned curve, the resulting curve will show how closely the watch has been adjusted to isochronism.

The 40 watches, previously mentioned, were tested for 36 hours after winding, while maintained in the horizontal, dial-up position and at a temperature of approximately $20 \mathrm{C}$. The curves of figure 7 were obtained from the results. Curves $A$ and $B$ represent watches of the 2 types of 1 make, and curves $C$ and $D$ those of the same types of another make. Each curve is the component curve for 10 similar watches. Curves $A$ and $C$ are for watches having the new vibrating assembly, and curves $B$ and $D$ are for watches having the old assembly.

The curves show very close agreement and close adjustment for $A, B$, and $D$, but a much greater variation for $C$. The shapes of the curves beyond the 24-hour point are of little consequence as watches are usually wound at intervals of, or reasonably close to, 24 hours. The new assembly does not appear to give any material advantage in the matter of isochronism over the old assembly. Watches of both typas may be adjusted to give about the same isochronal results.

The author wishes to acknowledge the valuable assistance rendered, in connection with the work reported in this paper, by H. I. Beehler, of the Hamilton Watch Co., and by A. B. Cook, of the Bureau of Standards.

Washington, February 24, 1934

\footnotetext{
7 Isochronism is the uniformity of rate as the mainspring unwinds.
} 